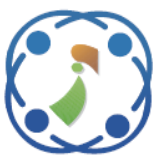

\title{
Peak to Average Power Ratio Reduction for Coherent Optical OFDM Systems Using Phase Rotation of The Sub-Carriers
}

\author{
Sinan Majid Abdulsatar ${ }^{1 *}$ \\ ${ }^{1}$ University of Technology, Iraq \\ * Corresponding author’s Email: sinansma@gmail.com
}

\begin{abstract}
Coherent Optical Orthogonal Frequency-Division Multiplexing (CO-OFDM) is widely used in optical communication systems, these systems suffer from many problems, including the high Peak-to-Average Power Ratio (PAPR). In this paper, a new method is proposed, it is based on Rotation Odd Constellation (ROC), this is done by dividing the subcarriers into two halves. The first half includes the Odd and the second consists of the Even subcarriers. A phase rotation is added to the first half, and no change is made in the second half. A simulation model has been accomplished by using Matlab platform and VPI transmission maker software to verify the efficiency of the proposed method and the improvment concerning Bit Error Rate (BER), Error Vector Magnitude (EVM) and Quality Factor $(\mathrm{QF})$. The results have been collected for128 subcarriers, 4QAM, 16QAM and bit rate of 10Gb/s, shows the best performance is obtained when $\pi / 4$ rad is the rotation angle.
\end{abstract}

Keywords: Orthogonal frequency division multiplexing, Peak-to-average power ratio, Quadrature amplitude modulation, Constellation rotation.

\section{Introduction}

The CO-OFDM system has several characteristics including good spectral efficiency and immunity against path losses that have enabled it to be used in high data rate systems applications [1-3]. The CO-OFDM system suffers from many problems, including the high PAPR. The problem of high PAPR in the CO-OFDM system increases the nonlinearity in the optical channel, which means an increase in Inter Carrier Interference (ICI), which also means that subcarriers lose their orthogonality, thus losing the transmitted signal [4].

In this paper, a new method is introduced and used for the first time in the Conventional COOFDM system. This method is called Rotation Odd Constellation (ROC) which is used to decrease PAPR of CO-OFDM.

The constellation rotation was used with encryption to increase diversity and to eliminate fading in the channels which rapidly changing as well as slow channels changing as in [5]. Also, The constellation rotation was used in a multi-antenna wireless transmission system to increase diversity and thus eliminate fading in channels as in [6,7], while the reshape of QAM constellation was used to reduce the PAPR and then connect it to the Selective Mapping (SLM) method or Partial Transmit Sequence (PTS) method to increase the PAPR reduction as in [8-10], these requires a large complexity for obtaining a PAPR minimization [11].

The rotation of QAM constellation is used in the OFDM system to reduce the complexity of the SLM method that used to mitigate PAPR through not need to send other information to the receiver as in[1214].

Finally, this paper suggested using rotated odd subcarrier to reduce PAPR for two mapping order 4QAM, 16QAM.

The effect of the rotation on PAPR and the system performance was investigated in the following section. This technique showed good results for PAPR reduction and enhanced the system performance for the system with 4QAM, 16QAM, 10Gbps, and 128 subcarriers. This paper organization has been carried out in five sections. 


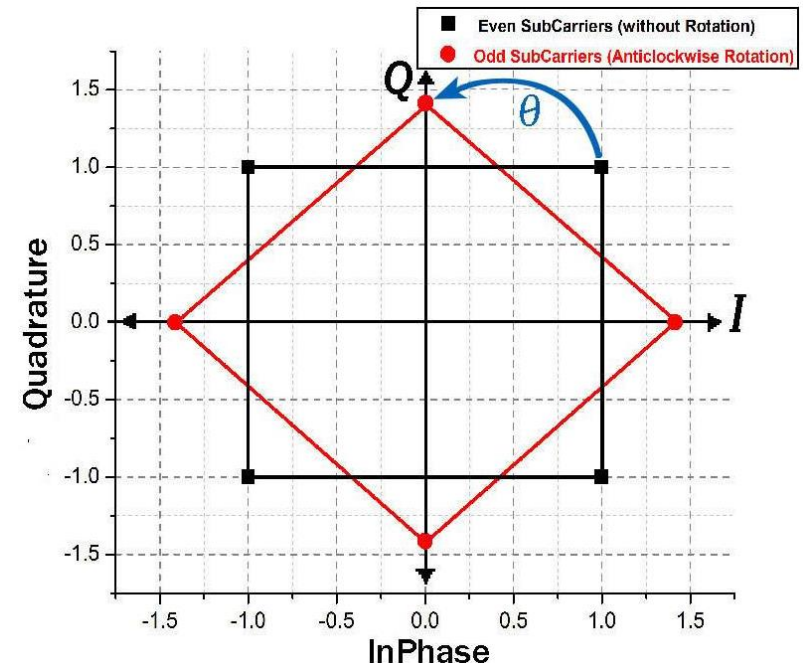

(a)

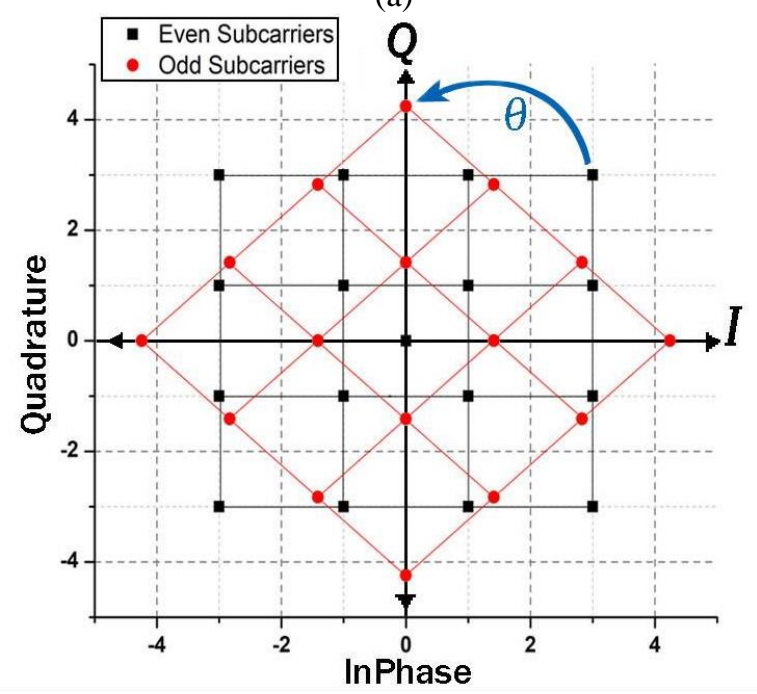

(b)

Figure. 1 Rotated QAM constellation: (a) 4QAMConstellation of odd and even subcarriers and (b) 16QAM constellation of odd and even subcarriers

Peak to Average Power Ratio Reduction for COOFDM Systems is introduced in section 1; section 2 describes the proposed technique principle. System setup is shown in section 3. Results and discussion are presents in section 4. Finally, I conclude my paper in section 5 .

\section{Proposed technique principle}

The suggested technique, which will be used to lessen PAPR of conventional CO-OFDM system, is based on dividing the subcarriers into two halves. The first half of subcarriers includes the Odd subcarriers and the second half of subcarriers consists of the Even subcarriers. After this stage, phase rotation is added to the first half, which includes the odd subcarriers and no change is made in the second half, as shown in Fig. 1.
The rotated odd subcarriers are used to reduce PAPR in all-optical OFDM for mapping order 4QAM only as in [15].

The contribution is to use ROC technique in conventional CO-OFDM to reduce PAPR for mapping order 4QAM, 16QAM and give a mathematical expression for finding $\mathrm{CCDF}$ for 4QAM and 16QAM.

Fig. 1 the odd subcarriers (red line) are rotated by an angle for 4QAM and 16QAM, while the even subcarriers (black line) remain without any rotation.

The OFDM Signal can be written as:

$$
x(t)=\sum_{k=-N / 2}^{N / 2-1} X_{k}(t) \exp \left(j \omega_{k} t\right)(1)
$$

$$
\omega_{k}=\frac{j 2 \pi k n}{N} \quad 0 \leq n \leq N-1
$$

Where the $X_{k}$ is the modulated signal on the $k_{t h}$ subcarrier, $N$ is the size of Inverse fast Fourier transform (IFFT) and $j=\sqrt{-1}$.

For the constellation rotation technique, the subcarriers have been divided into even, and odd subcarriers, and then the odd group is modulated with a rotated constellation in anti-clockwise, while the even subcarriers carry the symbols of $m Q A M$ with a regular constellation.

In the proposed technique, the OFDM signal field can be expressed as:

$$
\begin{aligned}
x(t) & =\sum_{\substack{k=-\frac{N}{2} \\
k \in \mathbb{O}}}^{\frac{N}{2}} X_{k}(t) \exp (j \theta) \exp \left(j \omega_{k} t\right) \\
& +\sum_{\substack{k=-\frac{N}{2} \\
k \in \mathbb{N}}}^{\frac{N}{2}} X_{k}(t) \exp \left(j \omega_{k} t\right)
\end{aligned}
$$

Where the first part $(\mathbb{O})$ represents the group of odd numbers as $\{-\boldsymbol{N} / \mathbf{2}+\mathbf{1},-\boldsymbol{N} / \mathbf{2}+\mathbf{3},-\boldsymbol{N} / \mathbf{2}+$ $5, \ldots, N / 2-1$, the second part (E) represents the group of even numbers as $\{-\boldsymbol{N} / \mathbf{2},-\boldsymbol{N} / \mathbf{2}+$ $2,-N / \mathbf{2}+\mathbf{4}, \ldots . N / \mathbf{2}\}$, and the rotation angle represented by $\boldsymbol{\theta}$. The normalized field envelope of a single $m Q A M$ subcarriers at CO-OFDM system transmitter can be written as [16]:

$$
X_{k}=\sqrt{\frac{P}{2}}\left(a_{k}+j b_{k}\right) \operatorname{rect}\left(\frac{t-k T_{s}}{T_{s}}\right)
$$

Where $(P)$ represents the power of subcarriers; and

$$
\operatorname{rect}(t)=\left\{\begin{array}{rr}
1 ; & \text { if } 0 \leq t<1 \\
0 ; & \text { otherwise }
\end{array}\right.
$$


It is noticeable that the complex amplitude is given by $A_{k}=a_{k}+j b_{k}$ for any number of $k$ and it is depended on the $m Q A M$ constellation.

The field of OFDM can be expressed as in the equation below after substituting Eq. (4) in Eq. (3):

$$
\begin{aligned}
x(t) & =\sqrt{\frac{P}{2}} \exp (j \theta) \sum_{\substack{k=1 \\
k \in \mathbb{C}}}^{\frac{N}{2}}\left|A_{k}\right| \operatorname{rect}\left(\frac{t-k T_{s}}{T_{s}}\right) \exp \left(j \omega_{k} t\right) \\
& +\sqrt{\frac{P}{2}} \sum_{\substack{k=-N \\
\frac{N}{2} \\
k \in \mathbb{E}}}^{\frac{N}{2}}\left|A_{k}\right| \operatorname{rect}\left(\frac{t-k T_{s}}{T_{s}}\right) \exp \left(j \omega_{k} t\right)
\end{aligned}
$$

The maximum of the optical field can occur when all subcarriers carry the same $m Q A M$ symbol and coherently merged. After some modification, the OFDM signal can be described by:

$$
\begin{aligned}
& |x(t)|=\sqrt{\frac{P}{2}}\left|\cos \left(\frac{\theta}{2}\right) \exp \left(j \frac{\theta}{2}\right)\right| \sum_{k=-N / 2}^{N / 2}\left|A_{k}\right|(7) \\
& |x(t)|=\sum_{k=-N / 2}^{N / 2} \sqrt{\frac{P}{2}} \cos \left(\frac{\theta}{2}\right)\left|A_{k}\right|(8)
\end{aligned}
$$

The field of $K_{t h}$ subcarriers is equal to:

$$
\left|x_{k}(t, \theta)\right|=\sqrt{\frac{P}{2}} \cos \left(\frac{\theta}{2}\right)\left|A_{k}\right|
$$

From the definition, the value of PAPR for the $x(t)$ signal is [17]:

$P A P R=\frac{\operatorname{Max}\left(|x(t)|^{2}\right)}{E\left[(x(t))^{2}\right]}$

Where the $E[*]$ represents the expectation operator. In the case of 4QAM the $\left|A_{k}\right|=\sqrt{2}$ because the real component is equal to imaginary component $\mathrm{I}=\mathrm{Q}=1$, but for $16 \mathrm{QAM}$ the $\left|A_{k}\right| \in\{\sqrt{2}, \sqrt{10}, 3 \sqrt{2}\}$.

For 4QAM the max power of $\mathrm{N}$ subcarriers is equal to:

$$
\max \left(\left|x_{k}(t, \theta)\right|^{2}\right)=P N \cos ^{2}\left(\frac{\theta}{2}\right)
$$

The average power is equal to:

$$
E\left[(x(t))^{2}\right]=\sqrt{N} \frac{P}{\sqrt{2}}(12)
$$

By putting Eqs. (12) and (11) in Eq. (10), yield:

$$
\operatorname{PAPR}(\theta)=\sqrt{2 N} \cos ^{2}\left(\frac{\theta}{2}\right)
$$

From Eq. (13) the factor that has an effective impact on Cumulative Distribution Function (CDF) is $\cos ^{2}$ $(\theta / 2)$, so the CDF of $($ PAPR $<x)$ for the suggested system is:

$$
\begin{aligned}
C D F & =\int_{0}^{x} \frac{y}{\cos ^{2}\left(\frac{\theta}{2}\right) \sigma^{2}} \exp \left[\frac{-y^{2}}{2 \sigma^{2} \cos ^{4}\left(\frac{\theta}{2}\right)}\right]^{N} \\
& =1-\exp \left[\frac{-x^{2}}{2 \sigma^{2} \cos ^{4}\left(\frac{\theta}{2}\right)}\right]
\end{aligned}
$$

Where $\sigma$ is the variance constant and is equal to 1.8. For substantial $N$-OFDM symbols, the equation given below considers PAPR as a random variable, where its apportionment is:

$P(P A P R \leq x)=\left[1-\exp \left[\frac{-x^{2}}{2 \sigma^{2} \cos ^{4}\left(\frac{\theta}{2}\right)}\right]\right]^{N}$

PAPR statistics is explained by the Complementary Cumulative Distribution Function (CCDF). For 4 QAM the CCDF illustrates the probability of OFDM signal when it exceeds a particular PAPR value, as follows:

$C C D F=1-\left[1-\exp \left[\frac{-x^{2}}{2 \sigma^{2} \cos ^{4}\left(\frac{\theta}{2}\right)}\right]\right]^{N}$

Now we will find the mathematical expression to PAPR and CCDF for the mapping order 16 QAM. For 16QAM, the max power of the OFDM signal with $N$ subcarriers is equal to:

$\max \left(\left|x_{k}(t, \theta)\right|^{2}\right)=P \cos ^{2}\left(\frac{\theta}{2}\right)$

The average power for 16QAM can be written as:

$$
\begin{gathered}
E\left[(x(t, \theta))^{2}\right]=\frac{0.25 P}{3 \sqrt{2}}+\frac{0.25 P}{\sqrt{2}}+\frac{0.5 P}{\sqrt{10}}=\frac{1.223 P}{\sqrt{2}}(18) \\
=1.223 \sqrt{N} \frac{P}{\sqrt{2}}
\end{gathered}
$$

By putting Eqs. (17) and (18) in Eq. (10), the PAPR for 16QAM equal to:

$$
\operatorname{PAPR}(\theta)=\frac{\sqrt{2 N}}{1.223} \cos ^{2}\left(\frac{\theta}{2}\right)
$$

The CCDF for the OFDM signal describes PAPR and indicates to the OFDM signal probability when it surpasses a specified PAPR value. The CCDF is expressed by the equation below: 
$C C D F=1-\left[1-\exp \left[\frac{-1.223 x^{2}}{2 \sigma^{2} \cos ^{4}\left(\frac{\theta}{2}\right)}\right]\right]^{N}$

\section{System setup}

The CO-OFDM system diagram that used in this section is contained three parts: transmission section, optical transmission channel and the receiver part as shown in Fig. 2. mQAMThe transmitter part is used to send the optical signal through the optical channel, the data generated by PRBS is modulated on the even and odd subcarriers. Odd subcarriers are modulated after the $m Q A M$ is rotated, and the even subcarriers are modulated as a standard mQAM mapping.

After that, the subcarriers are added coherently toset upthe optical OFDM signal which is modulated on the optical carrier using I-Q modulator to transmit it through the channel. The optical channel includes multi-span and loops of the optical fiber; every span contours $55 \mathrm{~km}$ of Standard Single Mode Fiber (SSMF), Dispersion Compensating Fibers (DCF) and ErbiumDoped Fiber Amplifier (EDFA) optical amplifier as illustrated in Fig. 3.

The SSMF is configured with $(16 \mathrm{ps} / \mathrm{nm} / \mathrm{km})$ of Chromatic Dispersion (CD) coefficient, the effective

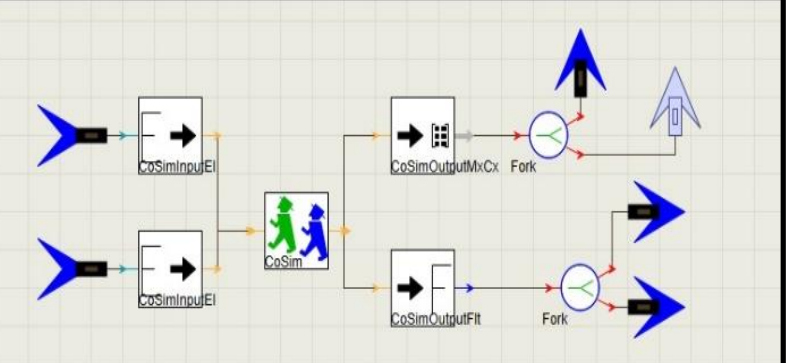

Figure. 2 OFDM decoder
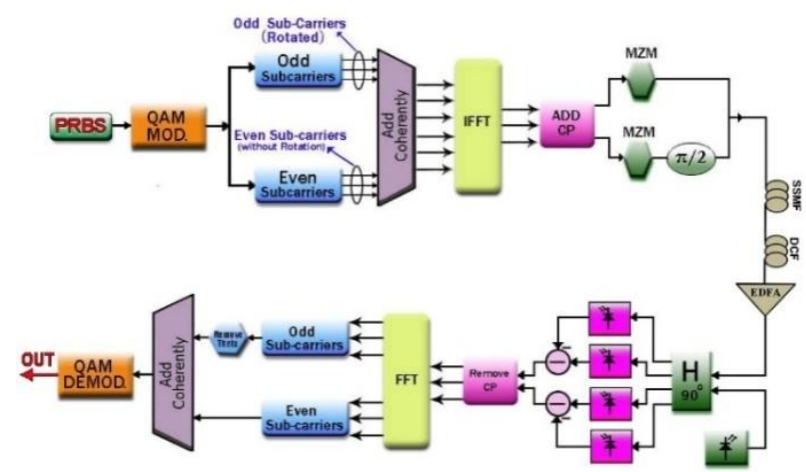

Figure. 3 Block diagram of CO-OFDM with ROC

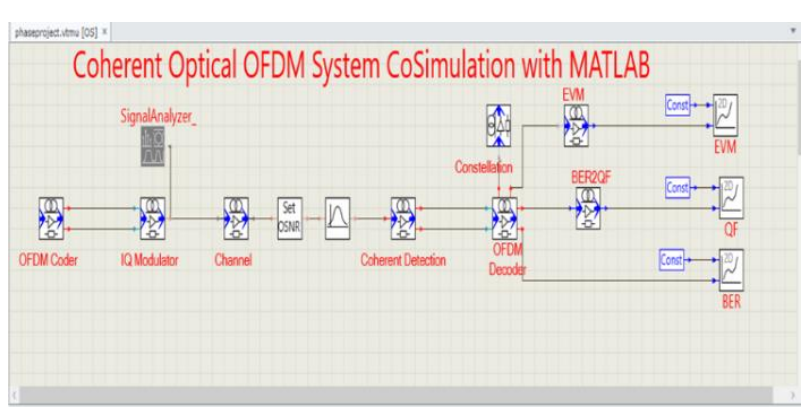

Figure. 4 Optical system setup

area of $\left(80 \mu \mathrm{m}^{2}\right)$ and attenuation coefficient $(\alpha)$ of $(0.2 \mathrm{~dB} / \mathrm{km})$. The DCF which has used to compensate the chromatic dispersion with $\alpha$ is equal to $(0.5$ $d B / \mathrm{km}$ ), and dispersion coefficient is equal to ($160 \mathrm{ps} / \mathrm{nm} / \mathrm{km}$ ). The EDFA with a noise figure is equal to $4 \mathrm{~dB}$ and gain of $13 \mathrm{~dB}$ to compensate losses in fibres and adjusted the launch power that used in SSMF and DCF. The CO-OFDM receptor is contained a coherent optical detector and OFDM detector.

\section{Results and discussion}

The System simulation has performed by Matlab Ver.R2014a and VPI transmission Maker ver. (9.5) Software. The simulation model was used to verify the qualification of the proposed techniqueconcerning PAPR reduction, as well as to improve system performance concerning BER, EVM, QF and constellation diagram for 4QAM and 16QAM. The simulation results have been collected for 128 subcarriers, 4QAM, 16QAM and bit rate of $10 \mathrm{~Gb} / \mathrm{s}$.

\subsection{Transmitter section}

Initially, the effect of the subcarriers that rotated on the PAPR value and the reduction amount obtained for the CO-OFDM system shown in Fig. 4 will be investigated. System parameters are brief in Table 1. Incontrast to the CCDF performance of the suggested method at $\theta=\pi / 6$ and $\theta=\pi / 4$ in radian versus standard system performance (no reduction of PAPR method) as it shown in Fig. 5 for 4QAM and 16QAM with subcarriers 128 .

For the system with 4QAM, Table 2. summarize the PAPR for the conventional OFDM $\theta=0, \theta=\pi / 6$ and $\theta=\pi / 4 \mathrm{rad}$. From the Table 2, the PAPR is enhanced by $0.8 \mathrm{~dB}$ when $\theta=\pi / 4 \mathrm{rad}$ and about $0.4 \mathrm{~dB}$ rad when $\theta=\pi / 6$ for $64,128,256,512,1024$ subcarriers.

For the system with 16QAM, Table 3. summarizes the PAPR for the original OFDM $\theta=0$, $\theta=\pi / 6$ and $\theta=\pi / 4 \mathrm{rad}$. From the table, the PAPR is 
minimized by $0.75 \mathrm{~dB}$ when $\theta=\pi / 4 \mathrm{rad}$ and about $0.35 \mathrm{~dB}$ when $\theta=\pi / 6 \mathrm{rad}$ for $64,128,256,512$ and 1024 subcarriers.

Table 1. System parameters

\begin{tabular}{|c|c|c|c|}
\hline \multicolumn{2}{|c|}{ Overall parameters } & \multicolumn{2}{|c|}{ Continues wave Laser } \\
\hline Bit-Rate & 10Gbps & Power & $5 \mathrm{dBm}$ \\
\hline Samples / bit & 2 & Frequency & $193.1 \mathrm{THz}$ \\
\hline Seq. length & 8192 & $\begin{array}{l}\text { Laserline- } \\
\text { width }\end{array}$ & $0.1 \mathrm{MHz}$ \\
\hline Sample Rate & 40Gbps & \multicolumn{2}{|c|}{ Single mode fiber \&DCF } \\
\hline $\begin{array}{c}\text { Number of } \\
\text { samples }\end{array}$ & 16384 & SMF Att. & $0.2 \mathrm{~dB} / \mathrm{km}$ \\
\hline mQAM & 4,16 & DCF length & $50 \mathrm{~km}$ \\
\hline Bits / symbol & 2,4 & $\begin{array}{c}\text { SMF } \\
\text { length }\end{array}$ & $5 \mathrm{~km}$ \\
\hline Constellation & Square & $\begin{array}{c}\text { DCF } \\
\text { Attenuation }\end{array}$ & $0.5 \mathrm{~dB} / \mathrm{km}$ \\
\hline \multicolumn{2}{|c|}{$\begin{array}{c}\text { Orthogonal FDM } \\
\text { modulator }\end{array}$} & DCF Disp. & $160 \mathrm{ps} / \mathrm{nm} / \mathrm{km}$ \\
\hline Subcarriers & 128 & $\begin{array}{c}\text { Number of } \\
\text { loop }\end{array}$ & 10 \\
\hline $\mathrm{CP}$ & $1 / 8$ & $\begin{array}{c}\text { SMF } \\
\text { Dispersion } \\
\end{array}$ & $16 \mathrm{ps} / \mathrm{nm} / \mathrm{km}$ \\
\hline
\end{tabular}

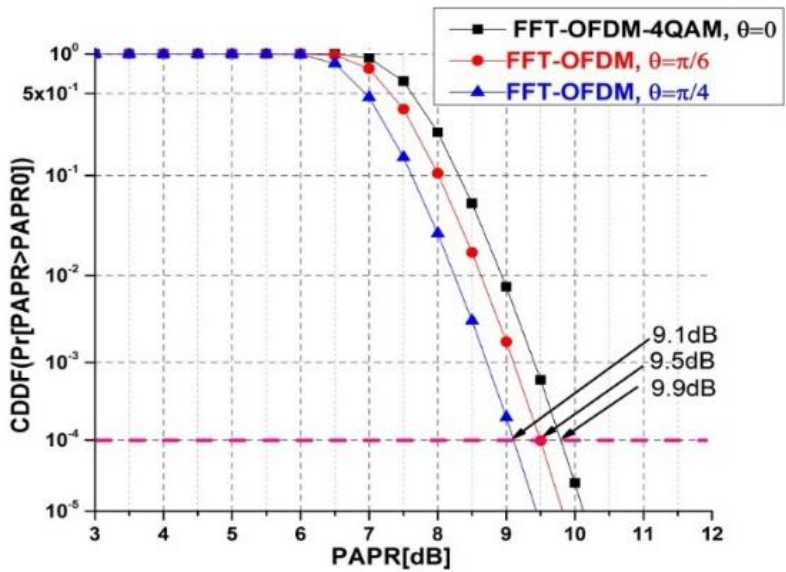

(a)

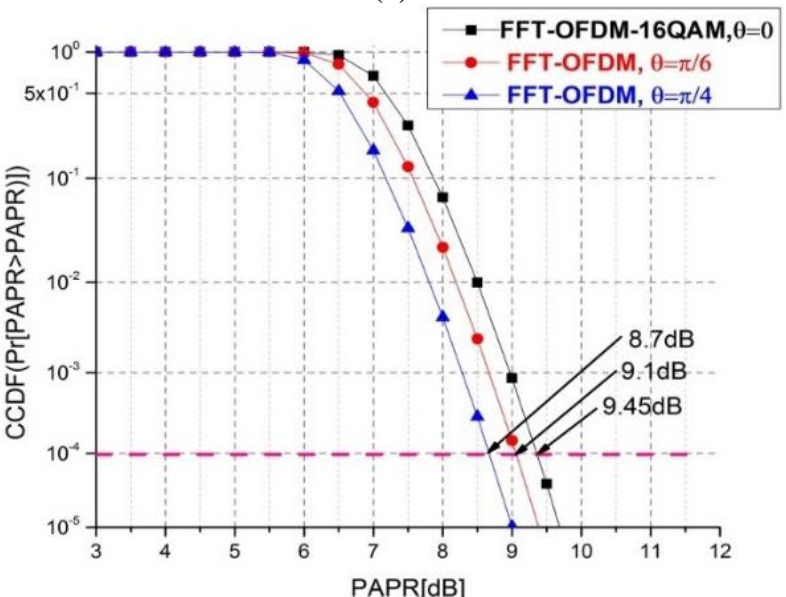

(b)

Figure. 5 CCDF versus PAPR: (a) CCDF vs. PAPR for 4QAM and (b) CCDF vs. PAPR for 16QAM
Table 2. PAPR for 4QAM at CCDF of probability $10^{-4}$

\begin{tabular}{||c||c||c||c||}
\hline No. Subcarrier & $\boldsymbol{\theta}=\mathbf{0}$ & $\boldsymbol{\theta}=\boldsymbol{\pi} / \mathbf{6}$ & $\boldsymbol{\theta}=\boldsymbol{\pi} / \mathbf{4}$ \\
\hline 64 & $9.8 \mathrm{~dB}$ & $9.4 \mathrm{~dB}$ & $9 \mathrm{~dB}$ \\
\hline 128 & $9.9 \mathrm{~dB}$ & $9.5 \mathrm{~dB}$ & $9.1 \mathrm{~dB}$ \\
\hline 256 & $10 \mathrm{~dB}$ & $9.6 \mathrm{~dB}$ & $9.2 \mathrm{~dB}$ \\
\hline 512 & $10.1 \mathrm{~dB}$ & $9.7 \mathrm{~dB}$ & $9.3 \mathrm{~dB}$ \\
\hline 1024 & $10.2 \mathrm{~dB}$ & $9.75 \mathrm{~dB}$ & $9.4 \mathrm{~dB}$ \\
\hline
\end{tabular}

Table 3. PAPR for 16 QAM at CCDF probability $10^{-4}$

\begin{tabular}{||c||c||c||c||}
\hline No. Subcarrier & $\boldsymbol{\theta}=\mathbf{0}$ & $\boldsymbol{\theta}=\boldsymbol{\pi} / \mathbf{6}$ & $\boldsymbol{\theta}=\boldsymbol{\pi} / \mathbf{4}$ \\
\hline 64 & $9.35 \mathrm{~dB}$ & $9 \mathrm{~dB}$ & $8.6 \mathrm{~dB}$ \\
\hline 128 & $9.45 \mathrm{~dB}$ & $9.1 \mathrm{~dB}$ & $8.7 \mathrm{~dB}$ \\
\hline 256 & $9.55 \mathrm{~dB}$ & $9.2 \mathrm{~dB}$ & $8.8 \mathrm{~dB}$ \\
\hline 512 & $9.65 \mathrm{~dB}$ & $9.3 \mathrm{~dB}$ & $8.9 \mathrm{~dB}$ \\
\hline 1024 & $9.75 \mathrm{~dB}$ & $9.4 \mathrm{~dB}$ & $9 \mathrm{~dB}$ \\
\hline
\end{tabular}

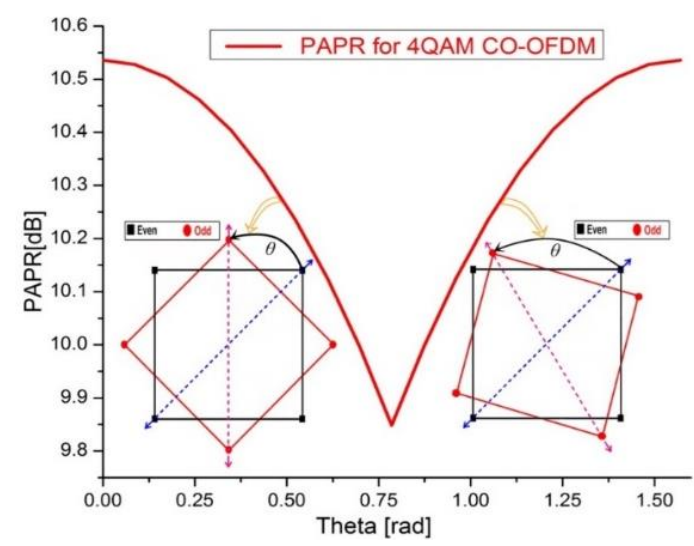

(a)

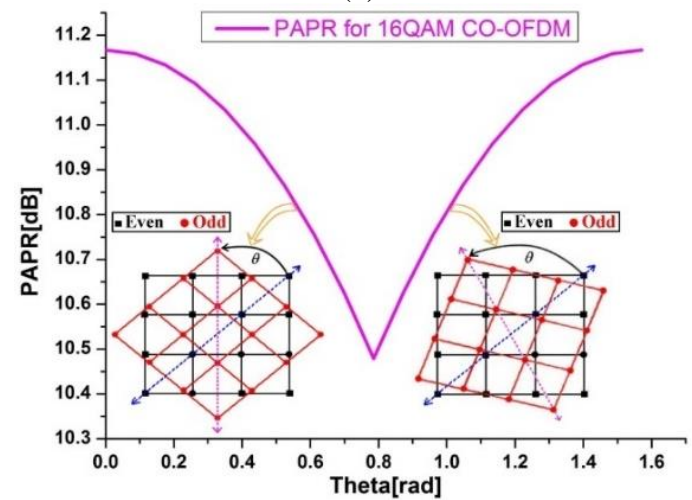

(b)

Figure. 6 PAPR with Different Value of Theta: (a) PAPR vs. Theta for 4QAM and (b) PAPR vs. Theta for 16QAM

The tables show that this approach is less sensitive to the number of subcarriers, and modulation order, so the system with 128 subcarriers was chosen to investigate its performance.

The PAPR with various angles of rotation is shown in Fig. 6 for 4QAM and 16QAM. increasing the angle of rotation between 0 and $\pi / 4$ radian is working to reduce PAPR. Nevertheless, when the rotation angle is increased for more than $\pi / 4 \mathrm{rad}$ that 
Table 4. Results of PAPR reduction methods at CCDF probability $10^{-4}$

\begin{tabular}{|c|c|c|c|c|}
\hline \multirow[t]{2}{*}{ Algorithm } & \multirow[t]{2}{*}{ Mapping } & \multirow[t]{2}{*}{ subcarrier } & \multicolumn{2}{|c|}{$\begin{array}{c}\text { PAPR } \\
\text { Reduction }(\mathrm{dB})\end{array}$} \\
\hline & & & Before & After \\
\hline SLM [18] & 4QAM & 64 & 11.2 & 9.9 \\
\hline PTS [18] & 4QAM & 64 & 11.2 & 9.7 \\
\hline Proposed Tech. & 4QAM & 64 & 11.2 & 9 \\
\hline Iterative Partial Transmit Sequence [19] & 4QAM & 256 & 11.2 & 10 \\
\hline Exponential Companding [20] & 4QAM & 256 & 11.2 & 9.8 \\
\hline Proposed Tech. & 4QAM & 256 & 10 & 9.2 \\
\hline
\end{tabular}
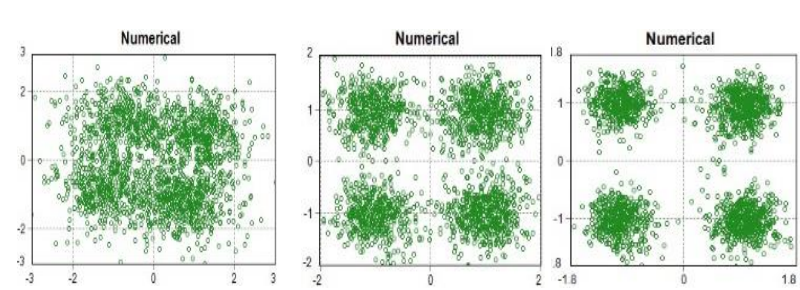

$\theta=0: 550 \mathrm{~km}$ $\theta=\pi / 6: 550 \mathrm{~km} \quad \theta=\pi / 4: 550 \mathrm{~km}$

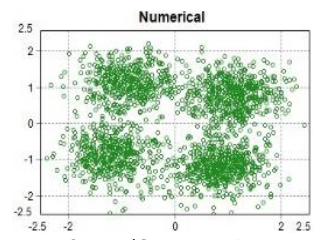

$\theta=\pi / 3: 550 \mathrm{~km}$

Figure. 7 Constellation diagrams with $\theta=\pi / 2: 550 \mathrm{~km}$

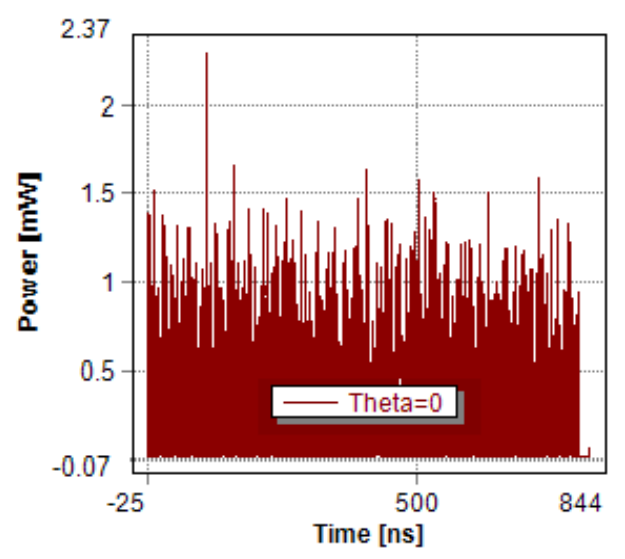

(a)

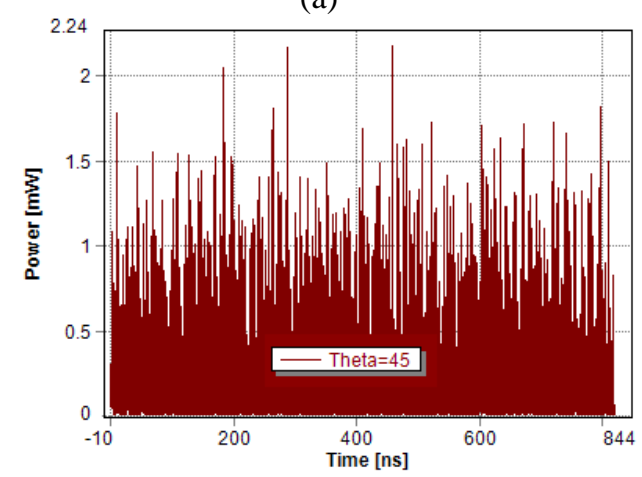

(b)

Figure. 8 Impact the rotation of the angle on the OFDM signals: (a) peak power for subcarriers for 4QAM at $\theta=0$ and (b) peak power for subcarriers for 4QAM at $\theta=\pi / 4$ also leads to increase PAPR because of the convergence between the even and odd constellation in next quarter. Therefore, a relation PAPR with $(\theta)$ is considered a periodic function and $\pi / 4 \mathrm{rad}$ is the optimum angle. Results of the simulation indicate that the PAPR drops from $10.5 \mathrm{~dB}$ to $9.8 \mathrm{~dB}$ for $4 \mathrm{QAM}$ and from $11.2 \mathrm{~dB}$ to $10.5 \mathrm{~dB}$ for $16 \mathrm{QAM}$ when $\theta$ increases from zero to $\pi / 4 \mathrm{rad}$ which is evident in Fig. 7 that represent the change in constellation diagram as the phase angle change.

A comparison results of the PAPR reduction methods shown at Table 4.

Fig. 8 shows the OFDM signal with a different rotation angle. Fig. 8 (a) illustrate the signal with a rotation angle $\theta=0$ for $4 \mathrm{QAM}$. It is indicated that the power for all subcarriers is same but the maximum peak equal to $2.37 \mathrm{~mW}$, while Fig. 8 (b) shows that peak power for a system with rotation angle $\theta=\pi / 4$ equal to $2.22 \mathrm{~mW}$ which refers to that any increase in the rotation of the angle leads to a decrease in signal peaks noticeably.

A comparison results of the PAPR reduction methods shown at Table 5 .

\subsection{Receiver section}

To show the impact of PAPR mitigation on the performance of the system and on the nonlinear impairments, Fig. 9 describes the EVM in dB which indicates the quality of the received signal variance versus the distance for the system with original OFDM signal and signal with rotation angles $\theta=\pi / 6, \pi / 4$ for 4 QAM CO-OFDM system. it is found that the EVM for a system with rotation angle $\pi / 4$ is better than the others when the distance increases. In the beginning, the EVMs are close to each other because the effect of nonlinear and phase noise is ineffective and the system performance is controlled by the optical amplifier.

This indicate that in order to obtained a BER equal to $1 \times 10^{-3} \mathrm{EVM}$ equal to $-10.624 \mathrm{~dB}$ for signal with $\theta=\pi / 4$ at a distance $400 \mathrm{~km}$ while for the 
Table 5. Comparison results of the PAPR reduction methods at CCDF probability $10^{-4}$

\begin{tabular}{|c||c||c||c|c||}
\hline \multirow{2}{*}{$\begin{array}{c}\text { Method } \\
\text { name }\end{array}$} & \multirow{2}{*}{ Mapping } & $\begin{array}{c}\text { Sub- } \\
\text { carrier }\end{array}$ & \multicolumn{2}{|c|}{$\begin{array}{c}\text { PAPR } \\
\text { Reduction(dB) }\end{array}$} \\
\cline { 4 - 5 } & & Before & After \\
\hline \hline Companding & & 10 & 9.65 \\
{$[21]$} & 16QAM & 512 & & \\
\hline $\begin{array}{c}\text { Proposed } \\
\text { Technique }\end{array}$ & & & 10 & 8.9 \\
\hline
\end{tabular}

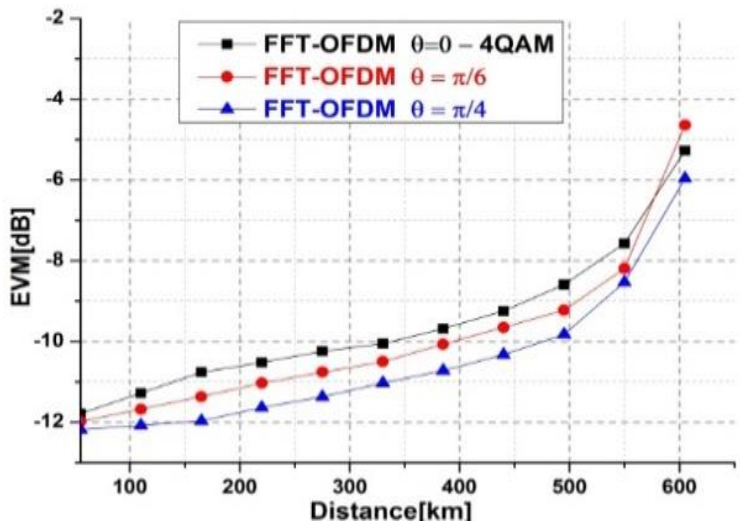

Figure. 9 EVM vs. Distance

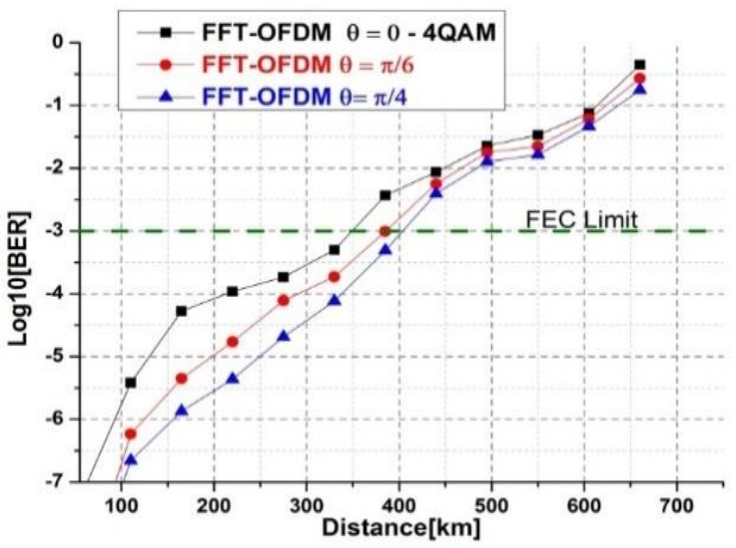

Figure. 10 BER vs. Distance

system with $\theta=\pi / 6$ EVM equal to $-10.065 \mathrm{~dB}$ at a distance $385 \mathrm{~km}$ and for system with $\theta=0$ EVM equal to $-9.854 \mathrm{~dB}$ at a distance $350 \mathrm{~km}$. From the above, there is an enhanced in EVM about $1.19 \%$ between them.

For the Fig. 10 which illustrated the relation between BER versus distance. As seen from the figure that BER for the system is changed continuously whenever the rotation angle changed. Maximum BER is obtained for the system with a rotation angle equal to $\theta=0$, whereas a minimum BER take place at $\theta=\pi / 4$. The BER is equal to $1 \times 10^{-3}$ at a rotation angle of $\pi / 4 \mathrm{rad}, 2.8 \times 10^{-2}$ at a rotation angle $\pi / 6 \mathrm{rad}$ and $2.4 \times 10^{-2}$ at a rotation

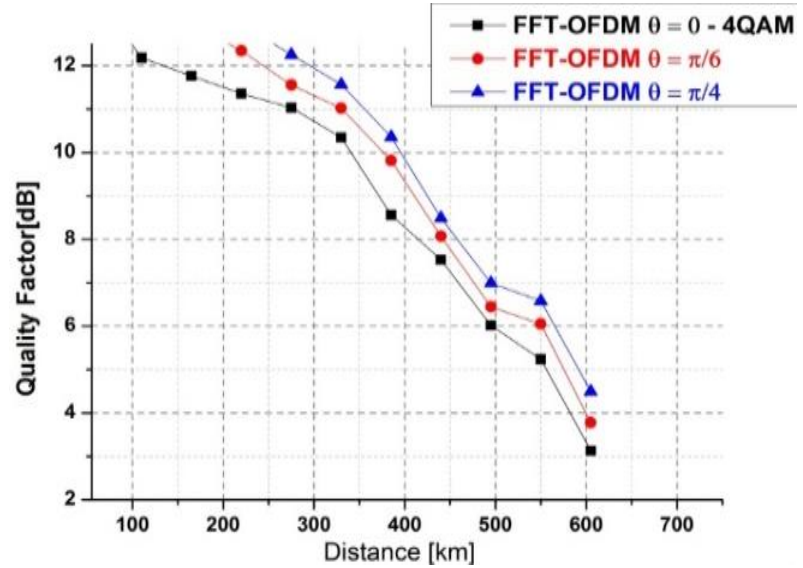

Figure. 11 Quality factor vs. Distance

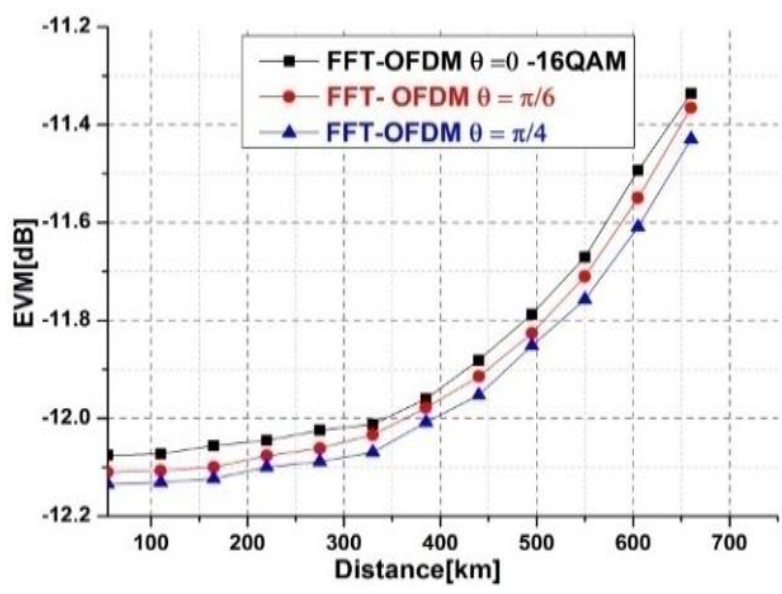

Figure. 12 EVM vs. Distance

angle equal to zero for the transmission distance of $400 \mathrm{~km}$. The BER has enhanced about -0.6 from the original signal.

Fig. 11 shows the $\mathrm{QF}$ in $\mathrm{dB}$ versus distance in $\mathrm{km}$. Also, from the figure, we concluded that the $\mathrm{QF}$ is varied as the rotation angle is varying.

As seen from the Fig. 11 that the QF is equal to $9.9 \mathrm{~dB}$ at rotation angle $\pi / 4 \mathrm{rad}, 9.4 \mathrm{~dB}$ at rotation angle $\pi / 6 \mathrm{rad}$ and $8.2 \mathrm{~dB}$ for the original signal at transmission distance equal to $400 \mathrm{~km}$. The $\mathrm{QF}$ enhanced about $1.7 \mathrm{~dB}$ and $0.5 \mathrm{~dB}$ at rotation angle equal to zero and $\pi / 6$ rad respectively.

From all of the above, system performance proves its improvement and gets better with the increase of the rotation angle which leads to mitigate the nonlinearity impairments of fibre because the power of the OFDM symbol is reduced with using the proposed technique.

Fig. 12 illustrated the relation between EVM, BER and QF against distance for 16QAM COOFDM system.

As seen from the Fig. 12, the system with $\theta=\pi / 4$ has a better EVM than $\theta=0$ or $\pi / 6$. At a distance of $440 \mathrm{~km}, \mathrm{EVM}=-11.88 \mathrm{~dB},-11.9 \mathrm{~dB}$ and $-11.96 \mathrm{~dB}$ 


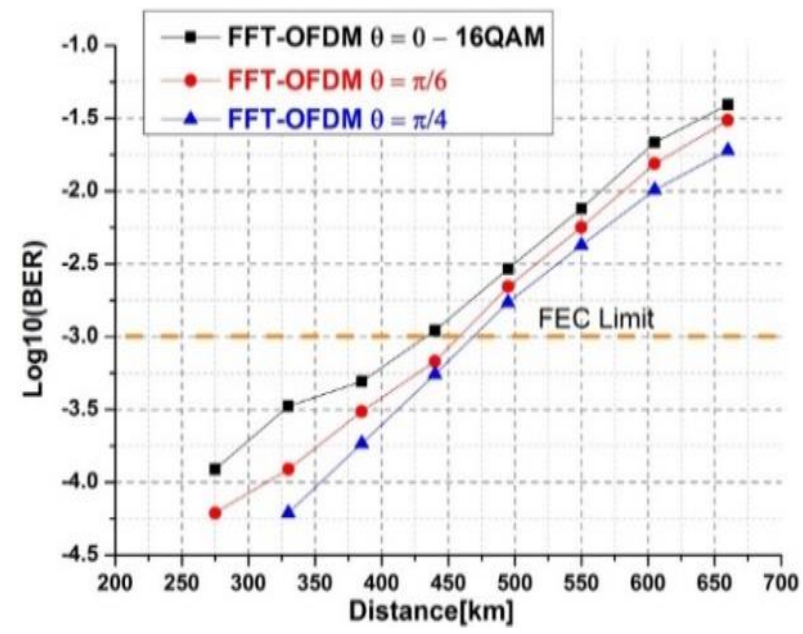

Figure. 13 BER vs. Distance.

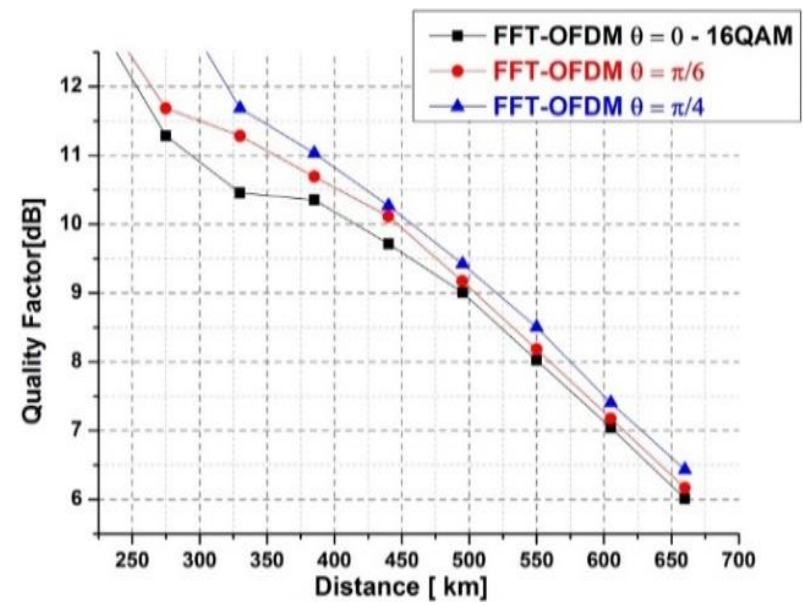

Figure. 14 Relation between quality factor and distance

for the system with rotation angle $0, \pi / 6$ and $\pi / 4 \mathrm{rad}$ respectively which means that EVM enhanced about 0.02 and $0.08 \mathrm{~dB}$ respectively.

Fig. 13 points that there is enhanced of BER. For the system with the rotation, angle $\pi / 4$ have BER equal to $1 \times 10^{-3}$ at a distance $475 \mathrm{~km}$, whereas for the system with rotation angle $\pi / 6$ the BER equal to $2.8 \times 10^{-2}$ and $2.6 \times 10^{-2}$ for the system with the original signal.

While Fig. 14 shows the QF for the system with rotation angle $\pi / 4$ is improved than the others. At a distance equal to $475 \mathrm{~km}$ the $\mathrm{QF}$ equal to $9.9 \mathrm{~dB}$, $9.5 \mathrm{~dB}$, and $9.3 \mathrm{~dB}$ for the system with rotation angle $\pi / 4, \pi / 6$, and $0 \mathrm{rad}$ respectively which means that there is improved in $\mathrm{QF}$ about $0.6 \mathrm{~dB}$ and $0.2 \mathrm{~dB}$ between them.

Fig. 15 (a) shows BER vs. OSNR for the signal with a rotation angle equal to zero, $\pi / 6$ and $\pi / 4$, where distance is fixed to $440 \mathrm{~km}$ for $4 \mathrm{QAM} \mathrm{CO}-$ OFDM. As seen from the figure that the system needs low OSNR to reach BER $1 \times 10^{-3}$. The OSNR is reduced by $0.5 \mathrm{~dB}$ from the system with rotation

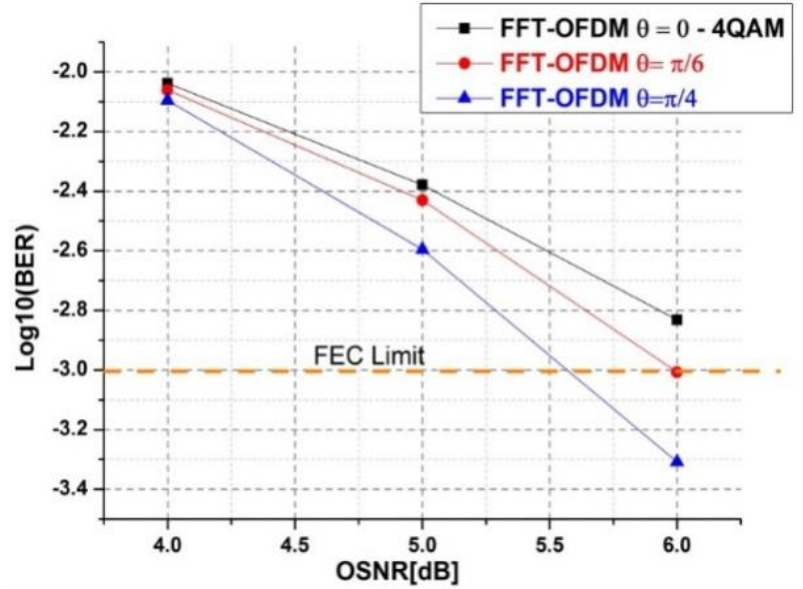

(a)

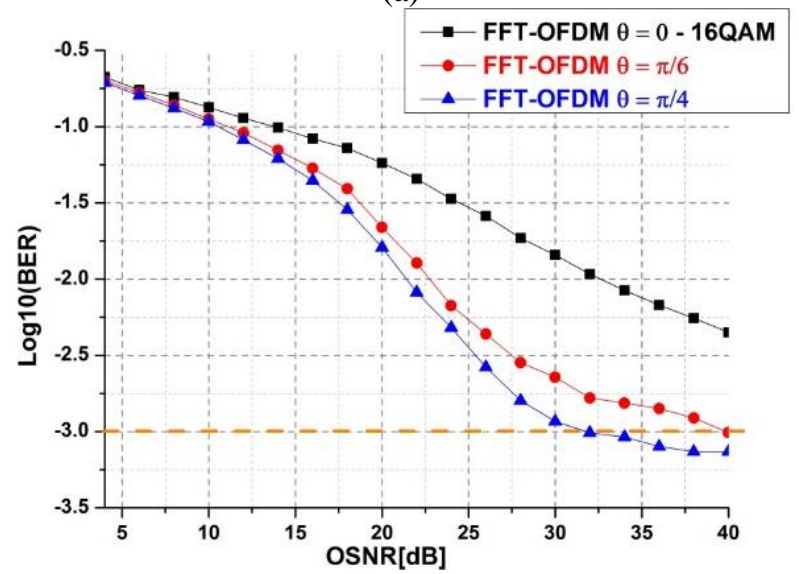

(b)

Figure. 15 (a) BER vs. OSNR for 4QAM CO-OFDM system and (b) BER vs. OSNR for 16QAM CO-OFDM system

angle $\pi / 6$ and more than $2 \mathrm{~dB}$ from the original system. Fig. 15 (b) illustrated the relation between OSNR and BER for 16QAM CO-OFDM system. The figure shows that the system with rotation angle $\pi / 4$ reaches BER $1 \times 10^{-3}$ at OSNR $30 \mathrm{~dB}$ which means it is reduced OSNR by $5 \mathrm{~dB}$ from the system with rotation angle $\pi / 6$ and more than $10 \mathrm{~dB}$ from the original signal.

Fig. 16 (a) represented the relation between QF and OSNR for 4QAM CO-OFDM system. At low OSNR, the QF is slightly different for three systems because the optical amplifier and DCF control the performance of the system. However, with higher OSNR, the difference between the QF variances becomes clearer because the proposed method reduces the nonlinear fiber impairments and the PAPR without any further distortion. There is an improvement of the quality factor of the system with rotation angle $\pi / 4$ by $0.5 \mathrm{~dB}$ and $0.75 \mathrm{~dB}$ from the system with rotation angle $\pi / 6$ and zero respectively. Fig. 16 (b) shows the relation between QF and OSNR for 16QAM CO-OFDM systems mentioned 


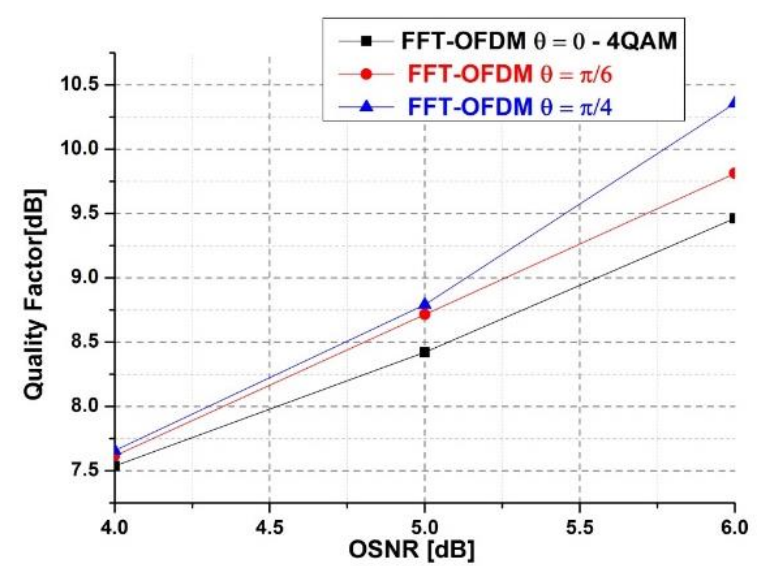

(a)

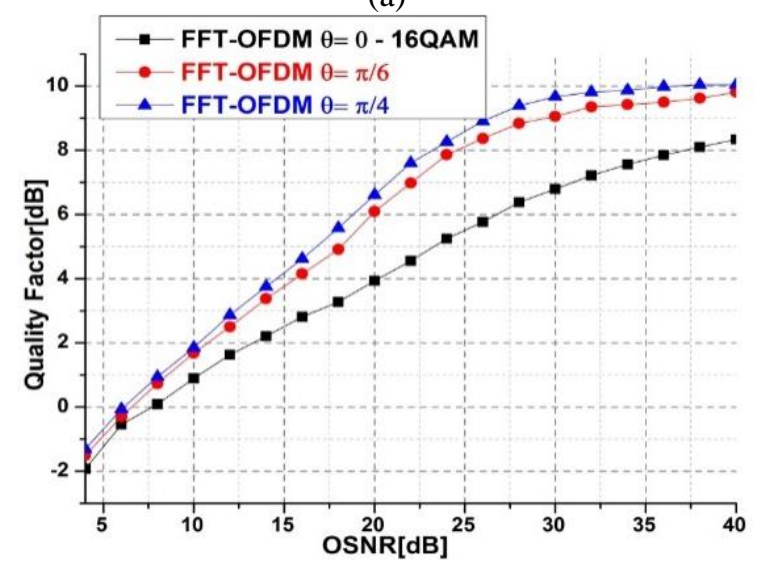

(b)

Figure. 16 (a) QF vs. OSNR for 4QAM CO-OFDM System and (b) QF vs. OSNR for 16QAM CO-OFDM System

in the previous section, the value of the quality factor of the three systems is close to each other when the OSNR value is small. When increasing the value of the OSNR, the difference between the quality factor of the three systems, especially for the system that uses the proposed method become clear. The QF for the system with rotation angle $\pi / 4$ is improved by $1 \mathrm{~dB}$ and $2 \mathrm{~dB}$ from the system with rotation angle $\pi / 6$ and zero respectively.

Fig. 17 (a) shows the relation between EVM and OSNR for 4QAM CO-OFDM, while Fig. 17 (b) for 16QAM CO-OFDM system. From the first figure of 4QAM, when the OSNR increases the EVM also increases. The system with angle $\pi / 4$ has a better EVM than the system with angle $\pi / 6$ or the original system. At OSNR equal to $10 \mathrm{~dB}$ EVM for the system with angle $\pi / 4$ improved about $0.25 \mathrm{~dB}$ than the original signal and about $0.1 \mathrm{~dB}$ than the system with angle $\pi / 6$. While for the CO-OFDM system of 16QAM the EVM for the system with angle $\pi / 4$ improved about $0.7 \mathrm{~dB}$ than the original one and $0.1 \mathrm{~dB}$ than the system with angle $\pi / 6$ at OSNR equal to $10 \mathrm{~dB}$, the Fig. 17 (b) is shown that.

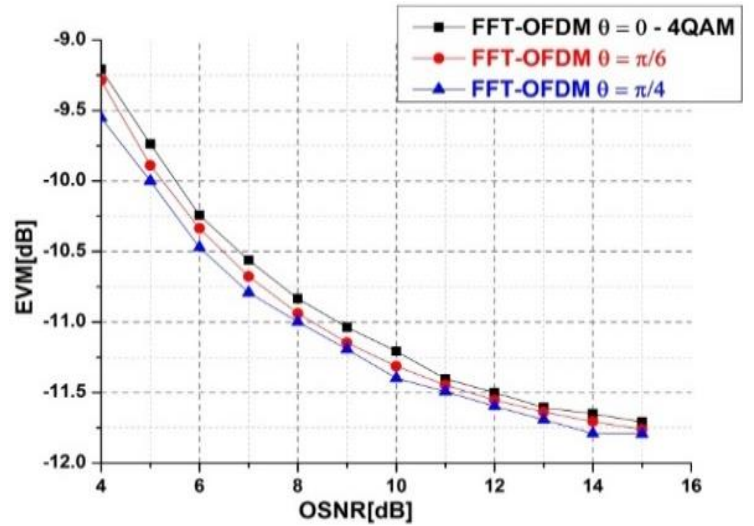

(a)

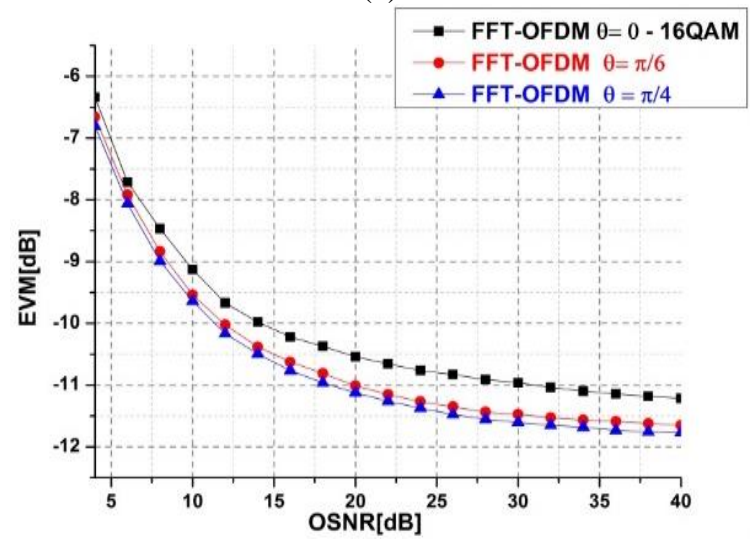

(b)

Figure. 17 EVM vs. OSNR: (a) 4QAM and (b) 16QAM

Finally, the relation between the BER and received power for the signal with and without the proposed method is drawn, attenuation with steps equal Sweep Attenuator has added $1 \mathrm{~dB}$.

Fig. 18 (a) and (b) show the outcomes for various phase rotation at $550 \mathrm{~km}$ for $4 \mathrm{QAM}$ and $385 \mathrm{~km}$ for 16QAM with launched laser power $5 \mathrm{dBm}$. As shown in Figs. 18 (a) and (b) whenever the received power increases the BER will be decreased.

Fig. 18 (a) with 4QAM and at a distance 550km, indicates that the system with the rotation angle $\pi / 4$ needs the minimum power about $-52 \mathrm{dBm}$ to reach the FEC limit, while the system with the rotation angle $\pi / 6$ and the system with the 0 rotation angle needs more than $-42 \mathrm{dBm}$ to reach FEC limits. For example, the BER for the system with $\pi / 4$ equals equal to $1 \times 10^{-3}$ at received power equal to $-52 \mathrm{dBm}$, while the BER for the system with the rotation angle $\pi / 6$ and the system with the 0 rotation angle equal to $1.8 \times 10^{-2}$ and $3.9 \times 10^{-1}$ respectively.

Fig. 18 (b) with 16QAM and a distance $385 \mathrm{~km}$, illustrates that the system with the rotation angle $\pi / 4$ needs the minimum power about $-48.5 \mathrm{dBm}$ to reach the FEC limit, while the system with the rotation angle $\pi / 6$ and the system with the 0 rotation angle 


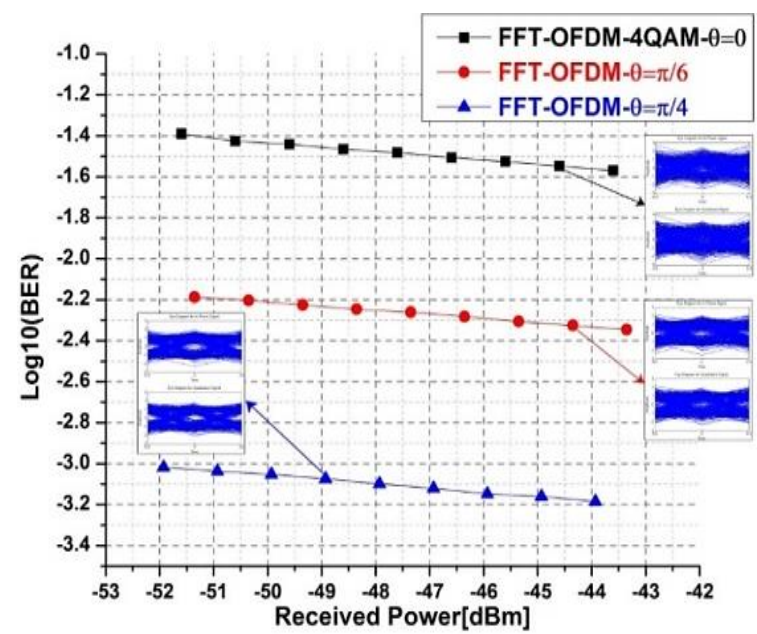

(a)

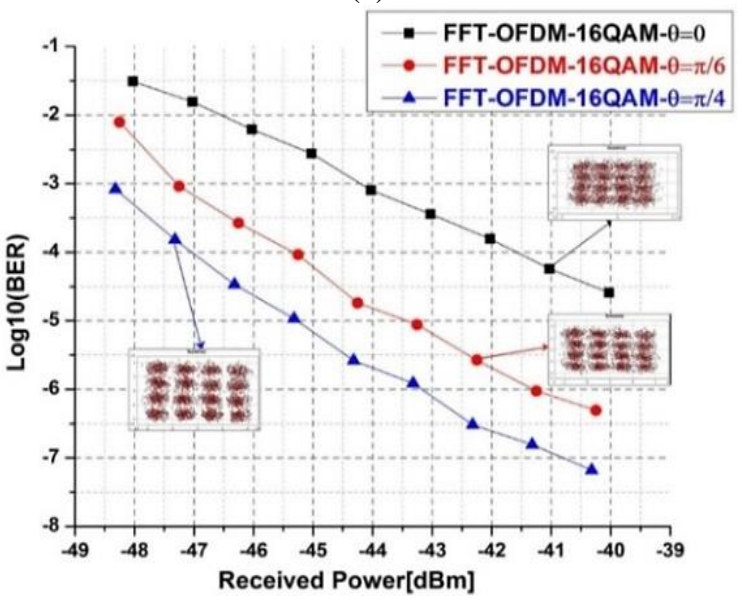

(b)

Figure. 18 Received power vs. BER: (a) 4QAM and (b) 16QAM

needs more than $-47.2 \mathrm{dBm}$ and $-44 \mathrm{dBm}$ respectively to reach FEC limits. For example, the BER for the system with $\pi / 4$ equals equal to $1 \times 10^{-3}$ at received power equal to $-48.5 \mathrm{dBm}$, while the BER for the system with the rotation angle $\pi / 6$ and the system with the 0 rotation angle equal to $1 \times 10^{-2}$ and $1.5 \times 10^{-1}$ respectively.

\section{Conclusions}

This paper touched upon a new technique which is used for CO-OFDM systems to reduce the nonlinear fibre impairments and reduction PAPR based on split the used subcarriers into two groups are even and odd subcarriers, then the odd subcarriers will be rotated immediately after QAM mapping in CO-OFDM systems has been modelled.

Reduction PAPR occurs when the QAM constellation is rotated for the odd group modulated, but the even group is modulated with the original QAM constellation.
The affected PAPR at an angle of rotation has been investigated. The impact of the reduction PAPR on the performance of the system has been accomplished by the simulation on the Matlab platform with VPI transmission maker software. CO-OFDM system is performed with 128 subcarriers, where each one has mapped by 4 and 16 QAM modulator.

Results show that the system performance depends on the difference in the angle between the constellation of odd \& even subcarrier, the best performance was obtained when $\pi / 4$ radisthe rotation angle. The proposed technique improved $\times 0.8 \mathrm{~dB}$ of PAPR and $\mathrm{CCDF}=1 \times 10^{-4}$ of $0.75 \mathrm{~dB}$ for 4QAM and 16QAM respectively and can mitigate the phase noise and fibre nonlinearity. Besides, the OSNR can be improved by the proposed system in comparison with conventional CO-OFDM has no PAPR reduction. The parameters were 4QAM with the rotation angle of $\pi / 4 \mathrm{rad}$ at the transmission distance is $440 \mathrm{~km}$, and the OSNR is improved by $2 \mathrm{~dB}$, for 16QAM at the transmission distance of $220 \mathrm{~km}$ the OSNR is reduced by more than $10 \mathrm{~dB}$ as well as the QF factor is improved by $0.75 \mathrm{~dB}$ for 4QAM and $2 \mathrm{~dB}$ for 16QAM. The efficiency of the ROC technique in this paper can be increased by integrating it with other PAPR reduction methods such as clipping, pre-coding or modified nonlinear methods of the discrete sliding norm transformer (DSNT). So new hybrid approaches may obtain more PAPR mitigation.

Furthermore, the ROC described in the $\mathrm{CO}$ OFDM system has dealt withwith a single polarization. For future work, the ROC method can be modeled for dual polarization Coherent Optical OFDM systems.

\section{References}

[1] J. Armstrong, "Orthogonal Frequency-Division Multiplexing for optical communications," Journal of Lightwave Technology, Vol. 27, No. 3, pp. 189-204, 2009.

[2] X. Chen, "Theoretical and experimental study on high spectral efficiency coherent optical OFDM systems", Ph.D thesis, University of Melbourne, 2012.

[3] X. Sun, "High data rate optical wireless communications based on ultraviolet band", M.Sc. thesis, University of KAUST, 2017.

[4] X. Yi, W. Shieh, and Y. Ma, "Phase noise effects on high spectral efficiency coherent optical Orthogonal Frequency-Division Multiplexing transmission", Journal of 
Lightwave Technology, Vol. 26, No. 10, pp. 1309-1316, 2008.

[5] Z. Qu, I. B. Djordjevic, and J. Anderson, "TwoDimensional Constellation Shaping in FiberOptic Communications", Applied Sciences, Vol. 9, No. 9, pp. 1889, 2019.

[6] V. M. DaSilva and E. S. Sousa, "Fadingresistant modulation using several transmitter antennas", IEEE Transactions on Communications, Vol. 45, No. 10, pp. 12361244, 1997.

[7] B. Vo, "Improved Spatial Modulation Techniques for Wireless Communications", Ph.D thesis, University of Saskatchewan, 2018.

[8] C. Li, T. Jiang, Y. Zhou, and H. Li, "A novel constellation reshaping method for PAPR reduction of OFDM signals", IEEE Transactions on Signal Processing, Vol. 59, No. 6, pp. 2710-2719, 2011.

[9] S.-J. Ku, "Low-complexity PTS-based schemes for PAPR reduction in SFBC MIMO-OFDM systems", IEEE Transactions on Broadcasting, Vol. 60, No. 4, pp. 650-658, 2014.

[10] F. Sandoval, G. Poitau, and F. Gagnon, "Hybrid peak-to-average power ratio reduction techniques: Review and performance comparison", IEEE Access, Vol. 5, pp. 2714527161, 2017.

[11] K. K. Kumar, A. Y. Babu, and B. T. Krishna, "Improved PTS Technique Based on Sub-Block Weighting Method of PAPR Reduction in OFDM Signals", International Journal of Intelligent Engineering and Systems, Vol. 10, No. 5, pp. 87-94, 2017.

[12] M. Sghaier, F. Abdelkefi, and M. Siala, "Efficient embedded signaling through rotated modulation constellations for SLM-based OFDM systems", In: Proc. of 2013 IEEE International Conference on Communications, pp. 5252-5256, 2013.

[13] S. P. Valluri, V. Kishore, and V. M. Vakamulla, "A New Selective Mapping Scheme for Visible Light Systems", IEEE Access, Vol. 8, pp. 18087-18096, 2020.

[14] W. Zou, T. Huang, J. Yuan, D. Wang, X. Li, and Z. Cheng, "Modified constellation reshaping method for PAPR reduction of PDM CO-OFDM based on a SLM algorithm", Applied optics, Vol. 58, No. 7, pp. 1800-1807, 2019.

[15] J. K. Hmood, K. A. Noordin, H. Arof, and S. W. Harun, "PAPR reduction in all-optical OFDM system using rotated constellation approach", Optical Fiber Technology, Vol. 25, pp. 88-93, 2015.
[16] H. Jassim Kadim, "Analysis and mitigation of nonlinear fiber impairments in high bit rate alloptical OFDM system/Jassim Kadim Hmood", Ph.D thesis, University of Malaya, 2016.

[17] D. Wulich, "Definition of efficient PAPR in "Reduction of of Peak to Average Power Ratio in orthogonal frequency division multiplexing using companding transform", IEEE Communications Letters, Vol. 9, No. 9, pp. 832-834, 2005.

[18] I. Tamlarasan, B. Swaminathan, and M. Murugaippan, "Improved fiber non-linearity mitigation despersion managed Optical Orthogonal Frequency Division Multiplexing link", Optic Comunications, Vol.385, pp.87-91, 2017.

[19] Y.-h. Liu, Z.-r. Tong, Y. Cao, W.-h. Zhang, and L. Li, "A novel joint technique for PAPR reduction in CO_OFDM systems", Optoelectronics Letters, Vol. 10, No. 4, pp. 277-280, 2014.

[20] A. Goel and P. Mathur, "Companding schemes for reducing Peak to Average Power Ratio in OFDM system: a review", Journal of Optical Communications, pp. 1-8, 2018.

[21] W. Ngah, S. Hashem, A. Abass, P. Varahrum, and S. Anas, "Reduction of PAPR in coherent optical orthogonal frequency division multiplexing using companding transform", In: Proc. of Reg.10 Symp., Kuala Lumpur, pp. 177180, 2014 